\title{
Automatic correction of the time step in implicit simulations of the stamping process
}

\author{
M.C. Oliveira*, L.F. Menezes \\ Department of Mechanical Engineering, University of Coimbra, Polo II-Pinhal de Marrocos, \\ 3030 Coimbra, Portugal
}

Received 5 August 2003; accepted 12 January 2004

\begin{abstract}
A specific aspect of simulations of stamping processes is the numerical method adopted to include the non-linearities associated with the frictional contact problem in large deformation plasticity. In fact, convergence problems are frequently encountered, especially when using a quasi-static implicit algorithm. One way of increasing the numerical robustness of these algorithms is to adopt an augmented Lagrangian method to incorporate the problem of contact with friction. The resulting non-linear system can be solved by a Newton -Raphson type method, for which a trial solution must be given to start the iterative equilibrium loop. The quality of this trial solution is determinant for the convergence of the iterative process. An automatic control of the size of the time step can improve this trial solution. In this study, a new size control for the time step, imposing restrictions not only on the increments of the state variables but also on the increments of the contact forces, is proposed. An example of a bending test is used to analyse the influence of this new limitation on the overall convergence behaviour of the fully implicit code DD3IMP.
\end{abstract}

(C) 2004 Elsevier B.V. All rights reserved.

Keywords: Finite element analysis; Implicit algorithms; Contact with friction

\section{Introduction}

The codes that use implicit algorithms to simulate deep drawing processes have general convergence difficulties, due to the complexity of the contact problem. However, it should be noted that the accuracy guaranteed for the state variables makes them very promising, encouraging the development of the implicit algorithms to improve their robustness. In the present paper the mechanical

\footnotetext{
${ }^{*}$ Corresponding author. Tel.: +351-239-790700; fax: +351-239-790701.

E-mail address: marta.oliveira@dem.uc.pt (M.C. Oliveira).
} 
model and the numerical methods used in the finite element code DD3IMP ${ }^{1}$, specially developed for the numerical simulation of the three-dimensional deep drawing process, are briefly described. The mechanical model takes into account the large elastoplastic strains and rotations that are associated with the forming process. To include the contact with friction, an augmented Lagrangian method is used, leading to a mixed formulation where the final unknowns are displacements and contact forces. The obtained non-linear system is solved by a Newton-Raphson method that can exhibit quadratic convergence in the vicinity of the solution [1-3].

The global convergence of the Newton-Raphson method, when applied to simulate the stamping process, depends mainly on parameters like: the numerical variables; the mechanical behaviour law; the friction coefficient; the geometry of the tools; the discretization of the metal sheet; the initial solution for the equilibrium iterative loop and the course of the deep drawing process, which can have distinct phases with different initial boundary conditions and strain paths. Although it is possible to identify the factors that influence the convergence, when divergence occurs it is very difficult to identify which of those parameters is the one responsible [3]. The results of the numerical tests undertaken showed that it is always possible to overcome the problems of convergence, acting on numerical parameters. They have also shown that, when divergence occurs, it generally results from difficulties in the contact strategy [4]. Modifying the numerical parameters, as the penalty coefficients or the limitations to the size of the time step, has no influence on the quality of the solution, making implicit methods highly sound and reliable [1-4].

In this study, the control of the initial solution of the iterative process is focused. A new set of limitations to the size of the time step is proposed, acting directly on the incremental contact forces that arise from the first trial solution. Numerical results of the bending of a metal sheet are shown, enhancing the advantages of a time step size controlled also by the trial contact forces.

\section{Mechanical model}

The mechanical model used in the numerical simulation of the stamping process takes into account the large elastoplastic deformations and rotations of a deformable body (sheet), in contact with rigid bodies (the forming tools). Since in the stamping process the elastic part of the transformation leads to small strains as compared with unity, the hypothesis of small elastic strains is applicable, regardless of the high elastic rotations that can occur [1-3]. The accuracy of the design solutions provided by numerical simulations strongly rely on the models used to describe the strong non-linearities resulting from the material and contact with friction. In the last years an enormous effort has been concentrated on the blank sheet material behaviour, concerning the improvement of both the behaviour laws and the initial yield locus description. The DD3IMP finite element code follows the same tendency and, nowadays, presents several isotropic and anisotropic constitutive models ( 7 isotropic/kinematic hardening laws and 8 yield criteria) [5,6]. The Coulomb law and the Signorini conditions are used to describe the contact with friction between the sheet and the tools. The evolution of the contact conditions is controlled by a mixed formulation, using an augmented Lagrangian approach.

\footnotetext{
${ }^{1}$ DD3IMP — contraction of Deep Drawing 3-D IMPlicit finite element code.
} 


\subsection{Principle of virtual velocities}

The region occupied by the deformable body is denoted by $\Omega_{0}$ and its boundary by $\Sigma_{0}$. On the part $\Sigma_{0 \mid 1}$ of the boundary the nominal stress vector is assigned, and on the complementary part $\Sigma_{0 \mid 2}$ the velocity vector is prescribed. This is the reference configuration in an updated Lagrangian scheme for the time interval $[t, t+\Delta t]$. For this reference configuration the principle of virtual velocities is given by the following equation [1]:

$$
\int_{\Omega_{0}} \mathbf{S}: \frac{\partial \delta \mathbf{v}}{\partial \mathbf{x}_{0}} \mathrm{~d} \Omega=\int_{\Sigma_{0 \mid 1}} \mathbf{s}^{*} . \delta \mathbf{v} \mathrm{d} \Sigma,
$$

valid for any virtual velocities field $\delta \mathbf{v}$ (with $\delta \mathbf{v}=0$ on the boundary $\Sigma_{0 \mid 2}$ ). $\mathbf{S}$ is the first PiolaKirchhoff stress tensor, $\mathbf{x}_{0}$ is the position vector of a generic point of the deformable body at time $t$. $\mathbf{s}^{*}$ expresses the external load corresponding to the prescribed nominal stress vector.

\subsection{Contact with friction}

In the absence of contact and friction equation (1) simply traduces the structural balance that can be written in the generic form:

$$
\mathbf{F}^{\text {int }}(\mathbf{u})-\mathbf{F}^{\text {ext }}=\mathbf{0} .
$$

$\mathbf{F}^{\mathrm{int}}(\mathbf{u})$ and $\mathbf{F}^{\mathrm{ext}}$ are the vectors of internal and external forces. The unknown of the problem is the displacement field $\mathbf{u}$. To include the problem of contact with friction, it is necessary to constrain the previous equation with the associated contact conditions. The Signorini conditions that define contact, and the Coulomb friction law, which imposes the contact status, are used. This is one of the main difficulties of the numerical simulation of the deep drawing process, since the boundary conditions are time-dependent. This dependency prevents a classic treatment of the problem, especially when using a fully implicit method. In fact, the inequations associated with the constraints imposed by the contact with friction induce strong non-linearities, requiring robust numerical methods for the solving of the global problem. The augmented Lagrangian approach has been successfully applied to solve this type of problem whenever it can be generalized to model the contact between a deformable body and a rigid obstacle of complex shape [7,8].

In the present case the augmented multiplier, $\boldsymbol{\sigma}$, is defined by the following equation [9]:

$$
\boldsymbol{\sigma}=\sigma^{\mathrm{n}} \mathbf{n}+\boldsymbol{\sigma}^{\mathrm{t}}=\left[\lambda^{\mathrm{n}}+p \mathrm{~d}^{\mathrm{n}}(\mathbf{u})\right] \mathbf{n}+\left[\lambda^{\mathrm{t}}+p \boldsymbol{\delta}^{\mathrm{t}}(\mathbf{u})\right],
$$

$\mathbf{n}(\mathbf{u})$ its the unit outward normal vector to the surface of the rigid obstacle, $\lambda^{\mathrm{n}}$ is the contact force normal to the rigid body and $\lambda^{\mathrm{t}}$ the tangential force at the contact point, $d^{\mathrm{n}}$ is the contact normal distance, $\boldsymbol{\delta}^{\mathrm{t}}$ is the tangential slip increment, and $p$ is a penalty coefficient. The contact and friction conditions are computed based on linear combinations of the kinematic and static variables, through the definition of the normal and tangential components of the augmented lagrangian, $\sigma^{\mathrm{n}}$ and $\boldsymbol{\sigma}^{\mathrm{t}}$, respectively. The use of this augmented multiplier corresponds to expanding the friction cone to positive values of the normal contact force $[7,8]$. In this way it is possible to formulate an augmented Lagrangian $\mathbf{L}^{p}(\boldsymbol{v}, \boldsymbol{\gamma})$ of virtual variables $\boldsymbol{v}$ and $\boldsymbol{\gamma}$, associated to the unknown vector fields $\mathbf{u}$ and $\lambda$, 
respectively. With this augmented Lagrangian the problem is exactly equivalent to the initial one, for all strictly positive values of the penalty coefficient $p$ and it can be written as follows $[1-3,8,10]$ :

$$
\left[\frac{\partial}{\partial \boldsymbol{v}}, \frac{\partial}{\partial \boldsymbol{\gamma}}\right] \mathbf{L}^{p}(\boldsymbol{v}, \boldsymbol{\gamma})=\left\{\begin{array}{c}
{\left[\mathbf{F}^{\mathrm{int}}(\mathbf{u})\right]+\left[\mathbf{F}^{\mathrm{equ}}(\mathbf{u}, \boldsymbol{\lambda})\right]-\left[\mathbf{F}^{\mathrm{ext}}\right]} \\
{\left[\mathbf{F}^{\mathrm{sup}}(\mathbf{u}, \boldsymbol{\lambda})\right]}
\end{array}\right\}=\mathbf{R}(\mathbf{u}, \boldsymbol{\lambda})=\mathbf{0} .
$$

The frictional contact Lagrangian $\mathbf{L}^{p}$ holds not only when there is contact, but also when the points are distant, due to the augmented contact pressure. This assures its global continuity [9]. $\mathbf{F}^{\text {equ }}(\mathbf{u}, \boldsymbol{\lambda})$ imposes the conditions of the contact with friction. $\mathbf{F}^{\sup }(\mathbf{u}, \boldsymbol{\lambda})$ takes into account the supplementary terms necessary for the control of the frictional contact boundary conditions allowing the determination of the contact forces.

\section{Finite elements}

Three-dimensional solid isoparametric finite elements are used in order to realistically define the sheet. The simultaneous contact on both sides is naturally taken into account, and it is possible to accurately calculate gradients across the thickness during the simulation. However, the isoparametric elements have a deficient behaviour when used to solve elastoplastic problems, since the full integration scheme causes the appearance of artificial hydrostatic stresses. To avoid this locking effect a selective reduced integration method is used [11,12].

After the spatial discretization of the deformable body, the system of non-linear equations (4) corresponds to six equations and six unknowns for each material point (node) of the deformable body in contact with the tools. The unknowns are the Cartesian components of the displacement vector $\mathbf{u}$ and of the contact force vector $\lambda$; leading to a mixed formulation in the pair $(\mathbf{u}, \lambda)$. The spatial discretization of the deformable sheet leads, after the assembly of the elemental matrices, to a global system obtained from the linearized form of equation (4):

$$
\left[\mathbf{K}_{A B}\right]\left\{\mathrm{d} \mathbf{u}_{B}, \mathrm{~d} \lambda_{B}\right\}=\left\{\mathrm{d} \mathbf{f}_{A}\right\},
$$

$A$ and $B$ are the node numbers, $\left[\mathbf{K}_{A B}\right]$ is the stiffness matrix. $\left\{\mathrm{d} \mathbf{u}_{B}, \mathrm{~d} \lambda_{B}\right\}$ is the displacement and contact force increment vector of the node $B$ of the deformable body and $\left\{\mathrm{d} \mathbf{f}_{A}\right\}$ is the "load" vector of the node $A[1,8,13]$. The mixed global system of linear equations is obtained considering the contact/friction operators that appear in Eq. (4). The contact is checked between a boundary of the deformable body and a rigid tool, described by Bézier surfaces $[1,8,13]$. The contact force $\lambda$ is then replaced by the contact force of node $A, \lambda_{A}$. This mixed system depends on the contact status of the node $A$, which influences the form of the contact operators $\mathbf{F}^{\text {equ }}$ and $\mathbf{F}^{\text {sup }}$. These contact operators also depend on the curvature of the parametric patches that define the tools, which directly influences the gradient of the normal vector at the contact point $[1,8,13]$. This combination of factors results in sets of equations very different for each node that conducts to an ill-conditioned, non-symmetric system. As the unknown nodal contact forces depend only on the degrees of freedom of the nodes to which they are associated, it is possible to transform the system in order to eliminate the unknown nodal contact forces [2]. In this way, a reduced system of linear equations is obtained for the nodal displacements, associated with an explicit definition of the contact nodal forces that can be 
expressed as:

$$
\begin{aligned}
& {\left[\mathbf{K}_{A B}^{\mathrm{red}}\right]\left\{\mathrm{d} \mathbf{u}_{B}\right\}=\left\{\mathbf{f}_{A}^{\mathrm{red}}\right\}} \\
& \mathrm{d} \lambda=f(\mathrm{~d} \mathbf{u}) .
\end{aligned}
$$

\subsection{Numerical methods}

The system $\mathbf{R}(\mathbf{u}, \boldsymbol{\lambda})=\mathbf{0}$ defined in Eq. (4) is a partial differentiable non-linear problem, without constrains. For its resolution it is possible to use a method of the Newton-Raphson type. In this algorithm, a single loop strategy is adopted for solving both non-linearities, resulting from the large elastoplastic deformation and the contact with friction. The reduced system of linear equations (6), obtained for each iteration of the algorithm, is solved with an iterative "Conjugate Gradient Squared" solver, with a "Level-k ILU" preconditioner [14].

To solve the problem using an implicit method, it is necessary to impose the equilibrium equations and the consistency condition on the configuration at time $t+\Delta t$, which is a priori unknown. To obtain a first solution for the incremental displacements stresses and contact forces, an explicit method can be used. This solution satisfies neither the variational principle nor the consistency condition, due to the non-linearity of the constitutive laws and to the kinematics of the problem. Therefore, this trial solution is corrected by using iterative method of Newton-Raphson type. So, each time increment of the simulation can be divided into two phases; the Predictor phase, which determines the explicit trial solution for each increment, and the Corrector phase, where this solution is improved by an implicit method [1-3].

\subsection{The tangent explicit method}

Since the formulation is quasi-static, the time increment can be replaced by the increment of any other monotonously increasing parameter, like the displacement of the tool that controls the forming process. In general, the stamping process can be divided in various phases where, in each one, the strain path is non monotonous. This means that the optimum incremental step size can change during the process. Assuming that the tool $M$ is controlling the phase, it is necessary to establish an initial incremental displacement $\mathrm{d} \mathbf{u}_{M}^{[N]}$ for that tool, for the configuration at time $t+\Delta t$, designated by $N$. In order to predict the evolution of the state variables and of the boundary conditions, the user imposes a trial value $\mathrm{d} \boldsymbol{\omega}_{\text {imp }}$ for each phase of the simulation, such as:

$$
\mathrm{d} \mathbf{u}_{M}^{[N]}=\mathrm{d} \omega_{\text {imp. }}
$$

With this imposed increment, it is possible to solve Eq. (4) with the explicit algorithm and obtain a first solution of the configuration $C^{[N, 0]^{\text {fic }}}$ for which the nodal contact forces and displacements are known. This allows the determining of the rotation, strain and stress increments. Since the integration method is explicit, all the calculated variables are proportional to $\mathrm{du}_{M}^{[N]}$. Then, the relation between the accepted trial increment $(\Delta)$ and this one $(\mathrm{d})$ is established by a weighting factor $r$. For a generic variable $\alpha$, this can be written as $[1-3,10]$ :

$$
\Delta \alpha=r \mathrm{~d} \alpha \text {. }
$$


The admissible value $r_{\min }$ of the coefficient $r$ is determined such that the change in the state of the elements and in the contact conditions during the increment does not induce too high modifications from the previous configuration. With this strategy, the increment size is not constant, and it is continuously adapted to the conditions of each increment, contributing to the improvement of the convergence of the iterative process in the Corrector phase. So, after obtaining the first explicit solution, this algorithm corrects the increment size, determining the value of $r$ for which, in each Gauss point of the deformable body finite element mesh:

- The largest absolute value of the incremental principal strains attains a prescribed limiting value $\Delta \varepsilon_{\max }$ :

$$
r_{\varepsilon}=\frac{\Delta \varepsilon_{\max }}{\max \left(\left|\mathrm{d} \boldsymbol{\varepsilon}_{1}\right|,\left|\mathrm{d} \boldsymbol{\varepsilon}_{2}\right|,\left|\mathrm{d} \boldsymbol{\varepsilon}_{3}\right|\right)} .
$$

- The Euclidean norm of the incremental rotations attains a prescribed limiting value $\Delta \omega_{\max }$ :

$$
r_{\omega}=\frac{\Delta \omega_{\max }}{|\mathrm{d} \boldsymbol{\omega}|} .
$$

- The largest value of the incremental normal and tangential stress to the yield surface are limited by a predefined surface:

$$
r_{T}=\frac{Y}{|\mathrm{~d} \mathbf{T}|\left(\frac{\cos \theta}{b}+\frac{\sqrt{1-\cos ^{2} \theta}}{a}\right)},
$$

with $\cos \theta=(\mathbf{Z}: \mathrm{d} \mathbf{T}) /(|\mathbf{Z}|:|\mathrm{d} \mathbf{T}|)$ with $\mathbf{Z}=\mathbf{M}:(\mathbf{T}-\mathbf{X})$. $\mathbf{M}$ is a fourth-order tensor characterizing the plastic anisotropy, $\mathbf{X}$ is the back stress tensor and $\mathbf{T}$ is the Cauchy stress, while $\mathrm{d} \mathbf{T}$ is its trial increment in the time step.

$\Delta \boldsymbol{\varepsilon}_{\max }, \Delta \boldsymbol{\omega}_{\max }, a$ and $b$ are user-defined parameters. Besides these limiting values, the course of the deep drawing process should also be considered. The end of a phase of the process is controlled by a displacement or force of a specific tool. To force the simulation to attain this phase limits, and validate a change of phase, a weighting factor $\left(r_{\text {pha }}\right)$ is calculated in each increment. Also, to have outputs for post-process purposes at predefined configurations a $\left(r_{\text {out }}\right)$ factor is also calculated. In order to exclude too small or too high incremental step sizes, a range of values $r \in\left[r_{\text {inf }}, r_{\text {sup }}\right]$ is also established. The admissible weighting factor $r_{\min }$ is then determined by the expression:

$$
r_{\min }=\max \left[\min \left(r_{\varepsilon}, r_{\omega}, r_{T}, r_{\text {pha }}, r_{\text {out }}, r_{\text {sup }}\right), r_{\text {inf }}\right] .
$$

\subsection{The implicit method}

After calculating the explicit trial solution of the increment $[t, t+\Delta t]$, the updated configuration $C^{[N, 0]}$ is given as a function of the incremental displacements, and frictional contact forces, $\Delta \mathbf{u}^{[0]}$ and $\Delta \lambda^{[0]}$. This configuration allows the calculation of the incremental strains and rotations, the integration of the constitutive laws, and the establishment of the first approximation to the stress and plastic strain fields at the end of the increment (instant $[t+\Delta t])$. To calculate the correct incremental displacements and contact forces, Eq. (4) is linearized with respect to the displacements in the neighbourhood of $C^{[N, 0]}$. This conducts to a system of linear equations, whose unknowns are 
$d u^{[1]}$ and $d \lambda^{[1]}$. This allows for the determination of a new approximation of the real increment $[1-3,10]$ :

$$
\left\{\begin{array}{l}
\Delta \mathbf{u}^{[N, 1]}=\Delta \mathbf{u}^{[0]}+\mathrm{d} \mathbf{u}^{[1]} \\
\Delta \lambda^{[N, 1]}=\Delta \lambda^{[0]}+\mathrm{d} \lambda^{[1]}
\end{array}\right.
$$

The new sheet configuration $C^{[N, 1]}$ and contact forces are then given by

$$
\left\{\begin{array}{l}
\mathbf{x}^{[N, 1]}=\mathbf{x}^{[N-1]}+\Delta \mathbf{u}^{[1]} \\
\lambda^{[N, 1]}=\lambda^{[N-1]}+\Delta \lambda^{[1]}
\end{array}\right.
$$

This iterative procedure is repeated until the norm of the out-of balance forces become lower than an imposed limit. Then the configuration at the end of the increment is updated and a new increment is started.

Although this method has quadratic convergence in the vicinity of the solution, numerical instabilities are frequent. The main difficulty of the numerical treatment is due to the fact that the contact problem introduces inequalities that lead to strong non-linearities. The quadratic convergence is restricted to a small differentiable region, which is characterised by the correct contact status of each point of the surface of the deformable body [3]. So, the first trial solution is of major importance to the subsequent convergence of the implicit algorithm.

\subsection{Control of the incremental contact force}

Figueiredo et al. [15] have demonstrated the existence and uniqueness of solution in a problem of contact with friction in thermoelasticity. These authors have shown that this problem has a local unique solution, imposing some restrictions on the friction coefficient, the contact force and eventually on the initial gap. Although this theoretical study is not on elastoplasticity problems, its conclusions can be very useful in the resolution of some convergence problems of the implicit algorithm presented above. In the numerical simulation of the deep drawing process, the friction coefficient cannot be changed, as it is a physical input data, and the gap is already controlled by the penalty parameter introduced in the augmented Lagrangian. So, a possible extrapolation of that work to the present algorithm can be done applying some limitations to the contact forces in the Predictor phase.

In the Predictor phase several parameters are controlled, but they are all associated to the internal variables (strain, rotation and stress). The contact forces are not restrained. However in some numerical simulations, the divergence of the algorithm can appear associated with incorrect values of the contact forces [4]. To solve this difficulty, we can restrain the incremental contact forces in order to assure the convergence of the algorithm without modifying the simulation process. This is only applied to the nodes in contact at the end of the previous configuration $C^{[N-1]}$, once no gap to contact changes are allowed during the Predictor phase. The explicit solution determines the fictitious contact forces $(\mathrm{d} \lambda)$. The real increment $(\Delta \lambda)$ is then forced to be limited to an ellipsoid according to the equation:

$$
\left(\frac{\mathrm{d} \lambda^{\mathrm{n}}}{c}\right)^{2}+\left(\frac{\mathrm{d} \lambda_{1}^{\mathrm{t}}}{d}\right)^{2}+\left(\frac{\mathrm{d} \lambda_{2}^{\mathrm{t}}}{e}\right)^{2}<B^{2},
$$

$\mathrm{d} \lambda^{\mathrm{n}}, \mathrm{d} \lambda_{1}^{\mathrm{t}}, \mathrm{d} \lambda_{2}^{\mathrm{t}}$ are the components of the incremental contact force normal to the sliding plane and in the sliding plane, respectively. $c, d$ and $e$ correspond to the different axes of the ellipsoid, which can 


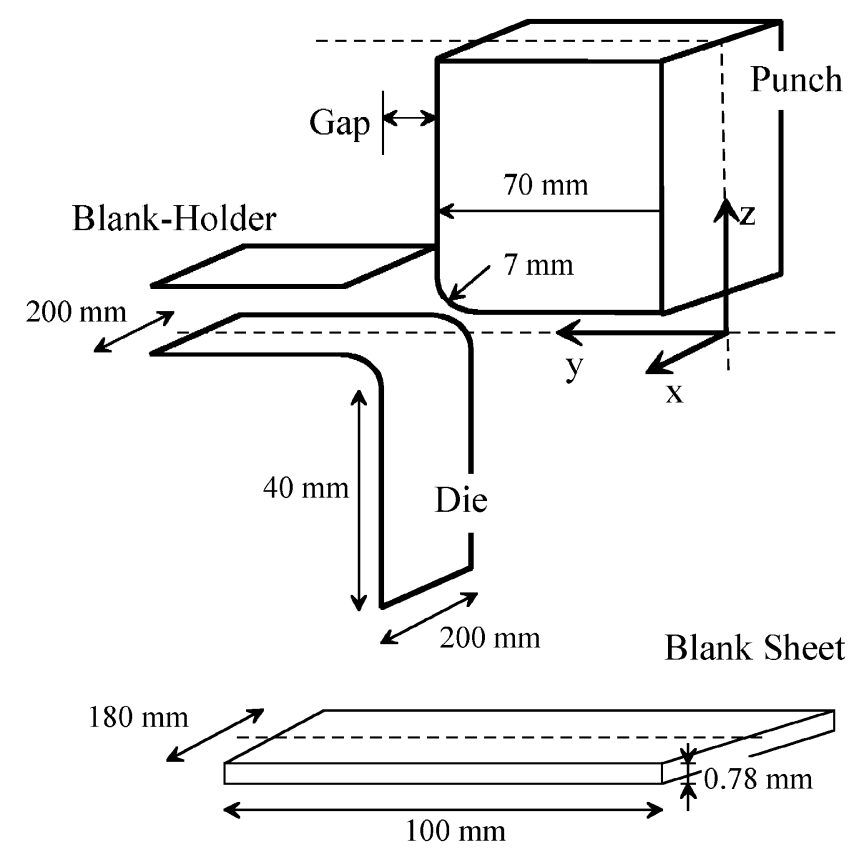

Fig. 1. Forming tools for the bending test.

take different values in order to control the components of the contact force vector differently. $B$ is used to normalize the value of the force components, since they can have different order values in the contact nodes. In this case $B$ is taken as the Euclidean norm, in each contact node, of the contact force in the configuration $C^{[N-1]}$ to avoid strong increases of $B$ during the new time increment:

$$
B=\left|\lambda^{[N-1]}\right| \text {. }
$$

A new weighting factor $r_{\lambda}$ is then calculated, for every node $A$ in contact, as follows:

$$
r_{\lambda_{A}}=\left(\sqrt{\frac{B^{2}}{\left(\mathrm{~d} \lambda^{\mathrm{n}} / c\right)^{2}+\left(\mathrm{d} \lambda_{1}^{\mathrm{t}} / \mathrm{d}\right)^{2}+\left(\mathrm{d} \lambda_{2}^{\mathrm{t}} / e\right)^{2}}}\right)_{A} .
$$

The final value of $r_{\lambda}$ will be the lowest value of $r_{\lambda_{A}}$. The new admissible weighting factor $r_{\min }$ is then determined by the expression

$$
r_{\min }=\max \left[\min \left(r_{\varepsilon}, r_{\omega}, r_{T}, r_{\lambda}, r_{\text {pha }}, r_{\text {out }}, r_{\text {sup }}\right), r_{\text {inf }}\right] .
$$

\subsection{Numerical results}

The efficiency of the proposed parameter was evaluated in the simulation of the bending of a mild steel sheet. The dimensions of the forming tools and initial blank sheet are described in Fig. 1. Due to the geometrical symmetry, only one half of the global structure is simulated. The mechanical properties of the mild steel considered are: $E=2.1 \times 10^{5} \mathrm{MPa} ; v=0.30$; Swift law parameters: $C=565 \mathrm{MPa} ; n=0.259$; Hill coefficients: $F=0.284, G=0.358, H=0.642, L=M=N=1.289$. 


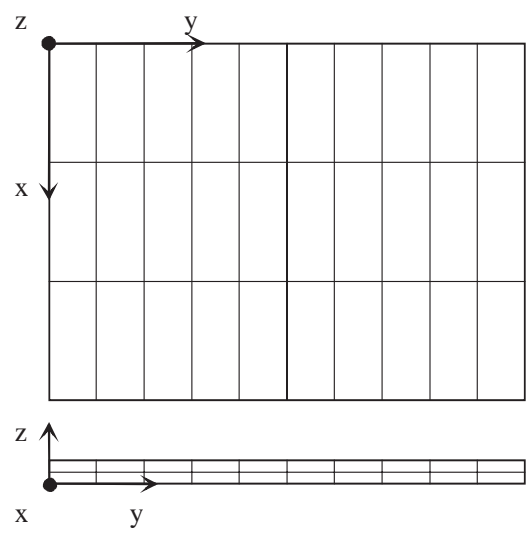

(a)

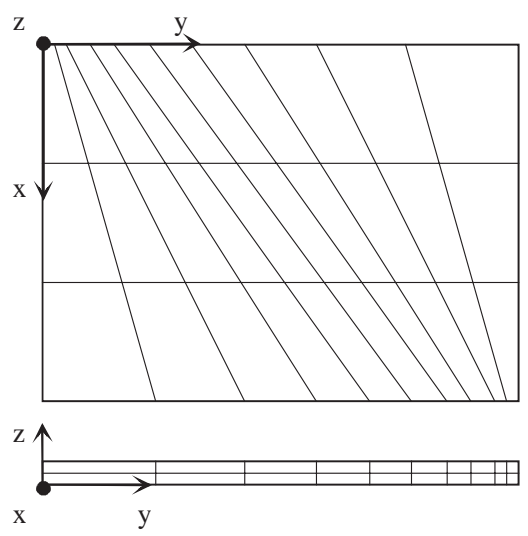

(b)

Fig. 2. Blank sheet meshes for the bending test.

Table 1

Limiting values for the determination of the admissible step increment

\begin{tabular}{llllll}
\hline$\Delta \varepsilon_{\max }$ & $\Delta \omega_{\max }$ & $a$ & $b$ & $r_{\text {inf }}$ & $r_{\text {sup }}$ \\
\hline 0.005 & $0.05^{\circ}$ & 3.0 & 1.0 & 0.001 & 1.0 \\
\hline
\end{tabular}

A friction coefficient of 0.144 is defined for the contact between the tools and the blank sheet. The tools are described by Bézier surfaces, three for the punch, three for the die and one for the blank-holder.

Since the goal of this study is to evaluate the influence of the limitation of the contact force increments on the global convergence of the implicit algorithm, some numerical difficulties are introduced. First, the blank holder force used is $22.6 \mathrm{kN}$, which minimises the sliding of the nodes in the flange, since is four times higher than the minimum theoretical value. Second, a high value of $5 \mathrm{~mm}$ is considered for the gap between the punch and the die. This value allows a higher rotation of the blank sheet in the die shoulder, introducing some difficulties in the contact detection in this region [16]. Finally, a rather coarse mesh is used to guarantee an easier interpretation of the results and to reduce the CPU time. Two different meshes, represented in Fig. 2, were tested. The main difference between them is the orientation of the elements in the sheet plane. The finite element meshes have 60 elements corresponding to a total of 88 nodes candidates for contact.

The chosen values for the increment size limitations on the strain, rotation and stress are taken from previous optimization studies [1], and they are presented in Table 1 . Several penalty coefficient values are tested $(p=100.0 ; 1000.0 ; 10000.0 ; 100000.0)$. They cover the range predicted for admissible values of this parameter $[1,8,17]$. It is important to clarify that all simulations are made with a constant value for $p$, for all nodes. In general during a numerical simulation with an implicit code, it is necessary to change the value of $p$ in order to overcome convergence problems. In this study, all simulations are assumed as stopped, as soon as, in an increment, the convergence is not attained after a pre-defined number of iterations. No attempt is made to continue the simulation. The convergence 


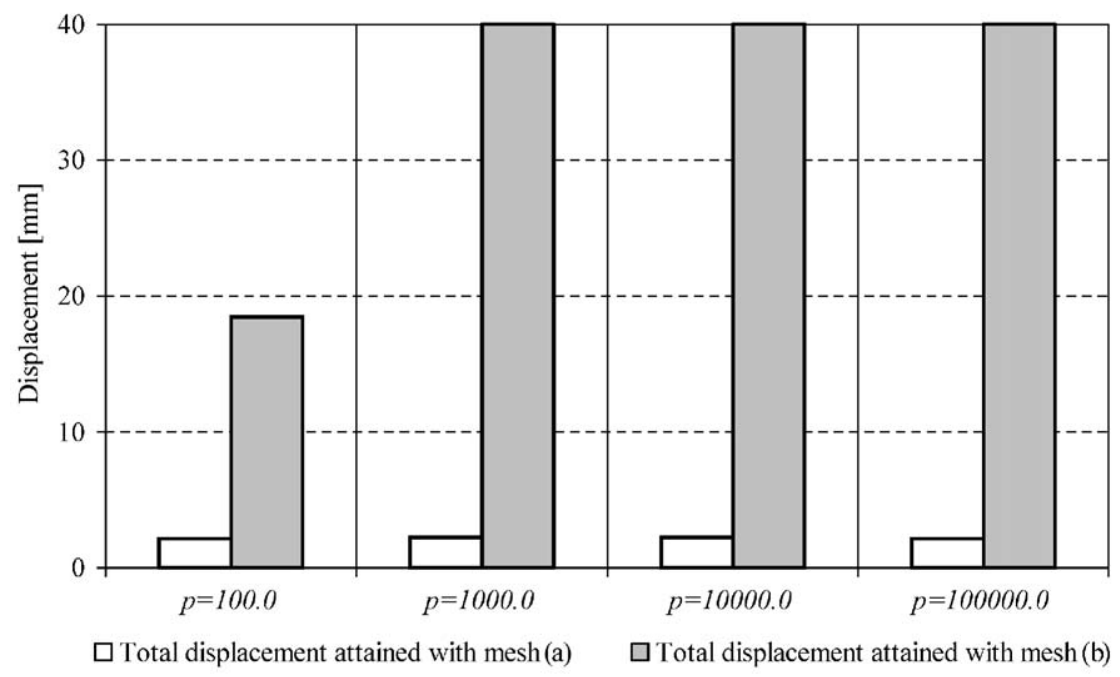

Fig. 3. Summary of the simulations performed with both meshes and with the step increment control defined by the parameters presented in Table 1.

criterion for each iteration $j$ is defined as $[1,8]$ :

$$
\left.C^{\text {Crit }}\right|^{j}=\frac{\|\mathrm{d} \lambda\|^{j-1}+\|\mathbf{R}\|^{j}}{\|\lambda\|^{j-1}},
$$

where $\mathbf{R}$ is the residual vector defined in Eq. (4) and the vector $\lambda$ is used in order to normalize the value of the convergence criterion in function of the contact forces involved in the process.

The first simulations performed were done with the limiting values presented in Table 1 and with no activation of the contact force control. These tests are used for comparison of the performance of the code, after the introduction of the new control parameter. The results are resumed in Fig. 3, which presents the total displacement of the punch achieved up to loss of convergence, for each fixed penalty parameter, $p$.

From these preliminary tests, it is possible to verify the importance of the mesh orientation in relation with the tools. In fact the contact detection for the presented problem is very difficult due to the reduced number of nodes in contact. During the first equilibrium iterations, in each time step, several nodes change their contact status. As the contact operator considered is only weakly differentiable, when contact status changes occur, this results in perturbations on the convergence $[3,13]$. In fact, only when the correct contact status is determined the convergence becomes quadratic. Mesh (a) has the same orientation of the tools. For the punch displacement of $2.0 \mathrm{~mm}$, all contact nodes along the same line are changing their contact status, as shown in Fig. 4. This effect influences the convergence of the process, whatever the penalty parameter. That is why all simulations stop at this punch displacement (Fig. 3). For mesh (b) this change of contact status is not so severe because it occurs for the different location nodes in different time increments. This leads to better results, and, for $p=1000.0,10000.0$ and 100000.0 the simulations with this mesh attain the process end, without any type of convergence problems. The results of mesh (a) might indicate that the penalty parameter has no influence, but since the penalty parameter acts directly on the contact status, it is 


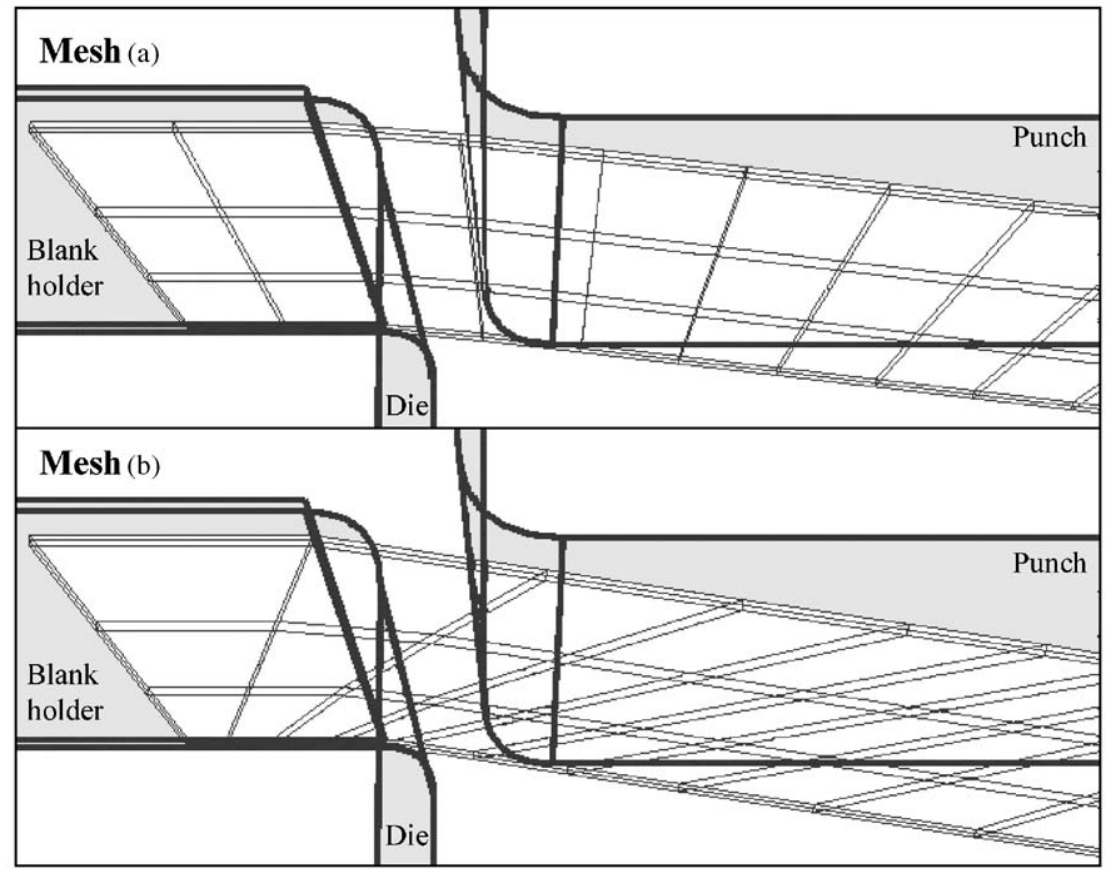

Fig. 4. Deformed shape and forming tools for both meshes, after a punch displacement of $2.0 \mathrm{~mm}$.

also an important factor. In fact, in using a small penalty parameter the augmented normal contact force is underestimated, which may lead to an incorrect "slip" contact status instead of a "stick" status. This is the reason why, for mesh (b), the use of a smaller penalty parameter does not result in a full simulation. It stops after a punch displacement of around $18 \mathrm{~mm}$.

With the introduction of the contact force control, two different strategies may be considered. If the parameters $c, d$ and $e$ (Eq. (17)) are equal, the ellipsoid becomes a sphere. The radius of this sphere is function of the value considered for the parameters, and of the Euclidean norm $B$ of the contact force in the previous step. Note that this normalized value $B$ corresponds to the norm of the effective contact force, and not to the increment of the contact force in the previous step. For $c$, $d$ and $e$ equal to 1.0 the radius of the sphere becomes equal to $B$, and the norm of the admissible increment for the contact force is equal to the norm of the contact force in the previous step. If the value for $c, d$ and $e$ is generically $c$, the limiting sphere presents a radius of $c B$. The problem is less restricted for $c$ larger then one. The influence of the value for the parameter $c$ is schematic represented in Fig. 5(a). Three different values for $c$ are tested: 0.1, 1.0 and 1000.0. These values were mixed with the values presented in Table 1 . The results of the simulations performed for both meshes are presented in Fig. 6.

The first conclusion that arises from these results is that the use of the contact force control conducts to better results for mesh (a), since it is possible to attain higher displacements without user intervention, in particular for small penalty values. The opposite occurs for mesh (b), where the best results are obtained for the higher penalty values. For mesh (a), the contact with the punch is performed in layers of elements in the direction Ox. So, for higher penalty values the nodes will 


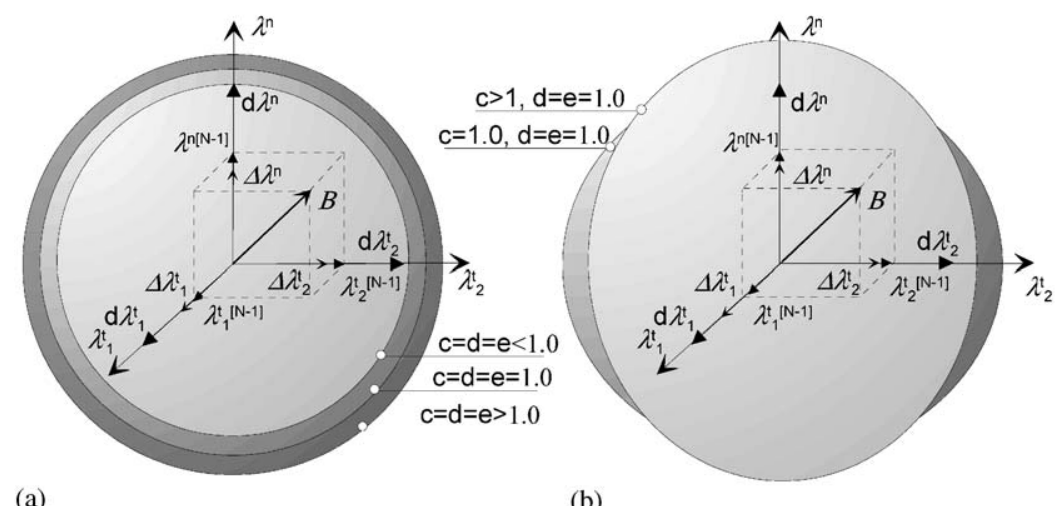

(a)

(b)

Fig. 5. Schematic representation of the limiting zone, induced by the contact force control: (a) $c, d$ and $e$ equal and (b) $c, d$ and $e$ different values.

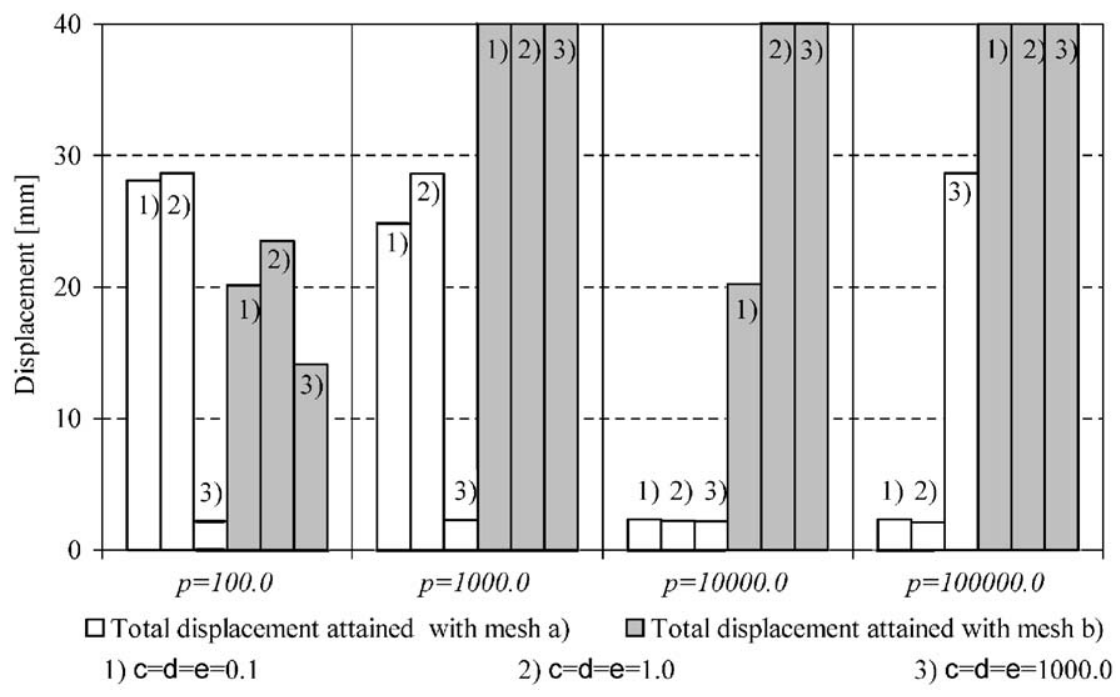

Fig. 6. Summary of the simulations performed with both meshes with the step increment control defined by the parameters $c, d$ and $e$ equal, and with the reference parameters of Table 1.

be attached with a 'stick' status to the punch, resulting in divergence problems. For mesh (b), there are always just a few nodes contacting with the punch. So, if the penalty value is small it is more difficult to maintain the contact and divergence problems can occur.

Comparing Fig. 6 with Fig. 3, it can be observed that for $p$ equal to 100.0 and $c$ equal to 1.0, the results obtained using the contact force control algorithm are significantly improved. This is also what occurs for the simulations performed with the penalty parameter equal to $100000.0, c$ equal to 1000.0 and mesh (a). In this situation, the larger value for the penalty parameter guarantees more contact nodes, and the lower control on the contact force allows a larger increment size. Another 


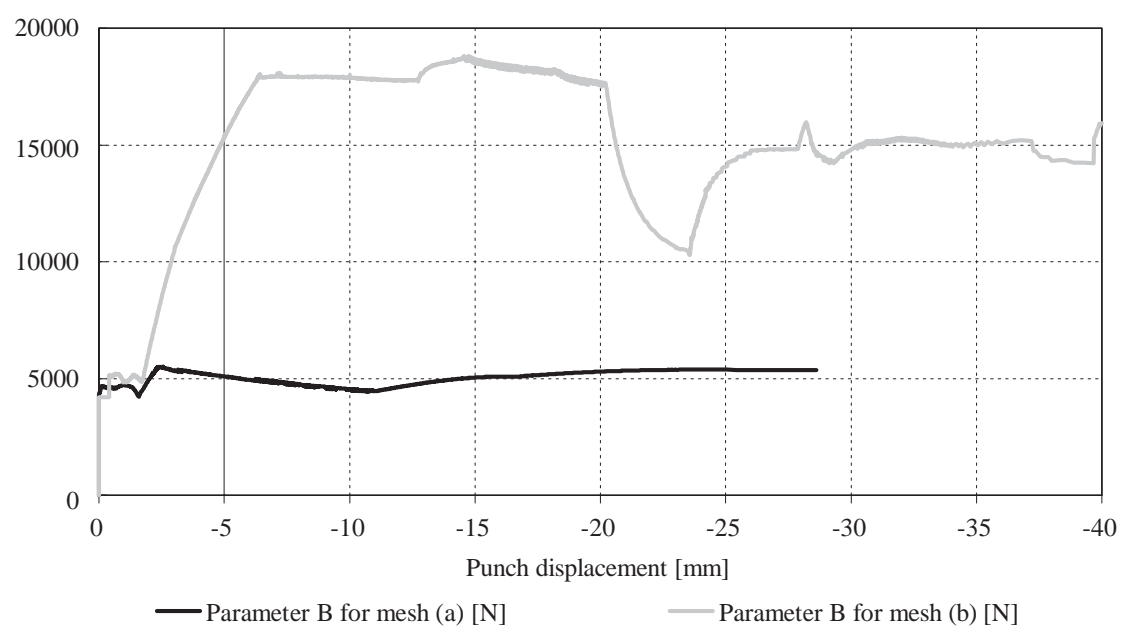

Fig. 7. Evolution of the normalizing parameter $B$ with the punch displacement.

important conclusion concerns the CPU time associated with the lower value for $c$. This limitation on the time step conduces to an enormous reduction of the parameter $r_{\min }$. The result is an enormous CPU time with no important improvements in the results. Taking as reference the mean CPU time for the simulations with mesh (b) that attained the final displacement of $40.0 \mathrm{~mm}$ with no contact force control, with the lower value of $c$ the CPU time increases $150 \%$. For these reasons, this lower value for the parameter $c$ was abandoned in the further simulations. In terms of the CPU time, comparing equal displacements, it is also possible to conclude that mesh (a) is very time consuming (about 3 times slower then mesh (b)). This occurs due to the characteristics of the mesh, since the large value of the punch/die gap implies a strong rotation of the elements in the die shoulder. The size of the elements of this mesh is too high to allow for rotation, conducing to very small time steps and high CPU times. This problem is well solved with mesh (b). Taking as reference the mean CPU time for the simulations with mesh (b) that attained the final displacement of $40.0 \mathrm{~mm}$ with no contact force control, with $c$ equal 1.0 the CPU time increases 7\%, and with $c$ equal 1000.0 the CPU time decreases around $2 \%$. Another important difference between the two meshes is the value of the normalizing parameter $B$. Since for mesh (a) there are more nodes in contact with the blank holder, this value is always smaller. In Fig. 7 the evolution of parameter $B$ is shown for a representative case of both meshes. The deformable node that controls the contact force parameter, during the deformation process is always a node in the flange. This results from the high blank holder force used, and that is the reason why the value of the parameter $B$ is dictated by the distribution of the blank holder force.

The second strategy in the contact force control algorithm is to consider an ellipsoid to determine the value of the contact force limitation for the time step. In contrast with the previous strategy, this apply different values for the parameters $c, d$ and $e$. In this study, only two cases are considered. The first assumes a higher control on the normal contact force $\lambda^{n}$, which results in an ellipse in the plane $\left(\lambda^{\mathrm{n}} ; \lambda_{1}^{\mathrm{t}}\right.$ or 2$)$ with the smallest axis in the direction $\lambda^{\mathrm{n}}$. In the other case, the control is mainly performed in tangential directions, which leads to an ellipse in plane $\left(\lambda^{n} ; \lambda_{1}^{t}\right.$ or 2$)$ with the smallest 




Fig. 8. Summary of the simulations performed with both meshes. The step increment control is performed with different values for the parameters $c, d$ and $e$ and the reference parameters of Table 1 .

axis in the direction $\lambda_{1}^{t}$ or 2 . Both strategies are schematically represented in Fig. 5(b). The summary of the performed simulations is presented in Fig. 8 .

The resume shown in Fig. 8, discloses the importance of controlling the time step with the contact force, in particular in situations where there are difficulties in the management of the contact with friction. These difficulties are very sensitive to the penalty parameter, but the contact force control allows for overcoming some of them. The introduction of the different contact force control parameters conduces, in some cases, to a reduction of the CPU time, especially when the tangential forces are more controlled. But this improvement depends strongly on the penalty parameter. Taking as reference the mean CPU time for the simulations with mesh (b) that attained the final displacement of $40.0 \mathrm{~mm}$ with no contact force control, for a higher control on the normal contact force the CPU time increases $3 \%$, and for a higher control on the tangential contact force the CPU time decreases around $8 \%$.

Finally, a new set of simulations was performed, fully relaxing all the time step control parameters, except the contact force ones. The results are presented in Fig. 9. Although these are not recommended procedures for performing a simulation, this group of simulations demonstrates that the contact force control combined with the appropriate penalty parameter can overcome some contact problems.

\section{Conclusions}

This paper describes the latest improvements on the numerical stability of the finite element code DD3IMP. A general description of the mechanical model and of the numerical methods used in the code is presented in order to depict the new strategy proposed. The code makes use of a fully implicit algorithm of Newton-Raphson type to solve in a single iterative loop the non-linearities 


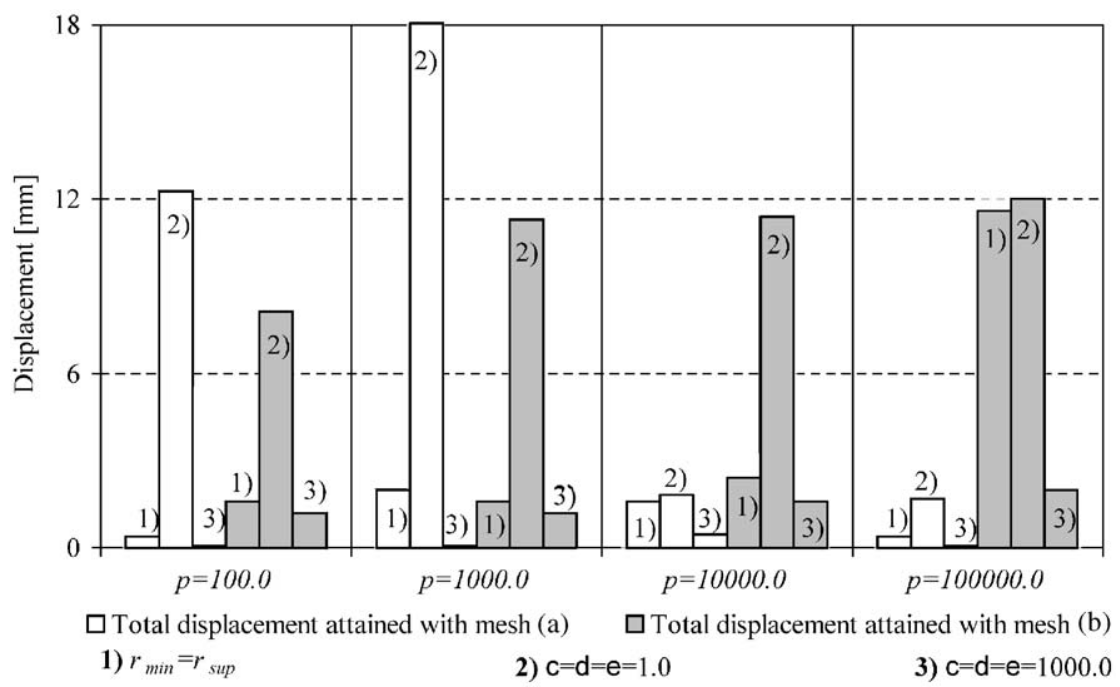

Fig. 9. Summary of the simulations performed with both meshes with the step increment control, where the parameters $c, d$ and $e$ are equal and the other parameters relax.

associated with the frictional contact and the elastoplastic mechanical behaviour of the material. The use of a Newton-Raphson method guarantees quadratic convergence of the iterative loop as long as the approximated first solution is not far from the equilibrium. To assure this proximity, control on the time increment is made by user-defined parameters. These parameters are usually based on the state variables. A new set of user-defined parameters was introduced in order to control the time step in function of the contact force increments.

This set of parameters allows for the overcoming of contact detection and status problems, especially in situations with a large percentage of nodes changing their contact status simultaneously. It is clear from the presented results that the force control parameter cannot, by itself, solve all the convergence problems that can arise during the simulation. Nevertheless, the association of this new strategy with the other set of control parameters improves the numerical stability of the simulations.

\section{Acknowledgements}

The authors are grateful to the Portuguese Foundation for Science and Technology (FCT) who financially supported this work, through the Program POCTI (Portuguese Government and FEDER).

\section{References}

[1] L.F. Menezes, Modelação tridimensional e simulação numérica dos processos de enformação por deformação plástica, Aplicação à estampagem de chapas, Ph.D. Thesis, Departamento de Engenharia Mecânica, Universidade de Coimbra, 1994.

[2] L.F. Menezes, C. Teodosiu, Improvement of the frictional contact treatment in a single loop iteration algorithm specific to deep-drawing simulations, in: J.C. Gelin, P. Picart, (Eds.), Proceedings of the NUMISHEET'99, the 
Fourth International Conference and Workshop on Numerical Simulation of 3D Sheet forming process, (Besançon, 1999), pp. 197-202.

[3] L.F. Menezes, C. Teodosiu, Three-dimensional numerical simulation of the deep-drawing process using solid finite elements, J. Mater. Process. Technol. 97 (2000) 100-106.

[4] M.C. Oliveira, Simulação numérica do processo de estampagem, Optimização de um algoritmo implícito, M.Sc. Thesis, Departamento de Engenharia Mecânica, Universidade de Coimbra, 1999.

[5] L.F. Menezes, S. Thuiller, P.Y. Manach, S. Bouvier, Influence of the work-hardening models on the numerical simulation of a reverse deep-drawing process, in: A.S. Khan, O. Lopez-Pamies, (Eds.), Proceedings of Plasticity'02: the ninth International Symposium on plasticity and its current applications, plasticity, damage and fracture at macro, micro and nanoscales, Neat Press, Maryland, 2001, pp. 331-333.

[6] J.L. Alves, M.C. Oliveira, L.F. Menezes, Influence of the yield criteria in the numerical simulation of the deep drawing of a cylindrical cup, in: E. Oñate, D.R.J. Owen, (Eds.), VII International Conference on Computational Plasticity COMPLAS 2003, Barcelona, 2003, pp. 158.

[7] P. Alart, A. Curnier, A mixed formulation for frictional contact problems prone to Newton like solution methods, Comput. Meth. Appl. Mech. Eng. 92 (3) (1991) 353-375.

[8] M.C. Oliveira, J.L. Alves, L.F. Menezes, Improvement of a frictional contact algorithm for strongly curved contact problems, Int. J. Numer. Meth. Eng. 58 (2003) 2083-2101.

[9] G. Pietrzak, A. Curnier, Large deformation frictional contact mechanics: continuum formulation and augmented lagrangian treatment, Comput. Meth. Appl. Mech. Eng. 177 (1999) 351-381.

[10] C. Teodosiu, H.L. Cao, Residual stresses after axisymmetric deep drawing, in: Proceedings of the 15th Biennial Congress, Controlling Sheet Metal Forming Processes, ASM International, Michigan, 1988, pp. 309-319.

[11] L.F. Menezes, C. Teodosiu, A. Makinouchi, 3-D solid elasto-plastic elements for simulating sheet metal forming processes by the finite element method, in: FE-simulations of the 3D Sheet Forming Processes in Automotive Industry, VDI Berichte Nr. 894. Verein Deutscher Ingenieure Editors, Düsseldorf, Germany, 1991, pp. 381-403.

[12] J.L. Alves, L.F. Menezes, Application of tri-linear and tri-quadratic 3-D solid FE in sheet metal forming process simulation, in: K. Mori, (Ed.), Proceedings of the NUMIFORM 2001, Seventh International Conference simulation of material processing: theory, methods and applications, Japan, 2001, 639-644.

[13] A. Heege, P. Alart, A frictional contact element for strongly curved contact problems, Int. J. Numer. Meth. Eng. 39 (1996) $165-184$.

[14] J.L. Alves, L.F. Menezes, Resolução de sistemas lineares esparsos em simulação 3-D do processo de conformação de chapa. Estudo da influência da numeração da malha, do precondicionador e do método iterativo, in: J.M. Goicolea, C. Mota Soares, M. Pastor, G. Bugeda (Eds.), Proceedings V Congresso de Métodos Numéricos en Ingenieria, Madrid, 2002, 95.

[15] L.M.N. Figueiredo, C.M.F. Leal, Numerical analysis of contact and friction problems in thermoelasticity, in: Actas da Escola de Elementos Finitos e Aplicações, I.M.N. Figueiredo (Ed.), Centro Internacional de Matemática, Coimbra, 1998, 1-33.

[16] M.C. Oliveira, L.F. Menezes, J.V. Fernandes, A numerical study on the influence of the punch/die gap in the deep-drawing process, in: J. Covas, (Ed.), Proceeding of the Second ESAFORM Conference on material forming, Guimarães, 1999, 109-112.

[17] P.W. Christensen, A. Klarbring, J.S. Pang, N. Stromberg, Formulation and comparison of algorithms for frictional contact problems, Int. J. Numer. Meth. Eng. 42 (1) (1998) 145-173. 